\title{
Satellite-based delivery of educational content to geographically isolated communities: a service based approach
}

\author{
Tacha Serif · Gheorghita Ghinea • \\ Lampros Stergioulas · Sherry Y. Chen · \\ Thanassis Tiropanis $\cdot$ Sofia Tsekeridou
}

Received: 4 December 2006/ Accepted: 8 May 2007

(C) Springer-Verlag London Limited 2007

\begin{abstract}
Enabling learning for members of geographically isolated communities presents benefits in terms of promoting regional development and cost savings for governments and companies. However, notwithstanding recent advances in e-Learning, from both technological and pedagogical perspectives, there are very few, if any, recognised methodologies for user-led design of satellitebased e-learning infrastructures. In this paper, we present a methodology for designing a satellite and wireless based network infrastructure and learning services to support distance learning for such isolated communities. This methodology entails (a) the involvement of community members in the development of targeted learning services from an early stage, and (b) a service-oriented approach to learning solution deployment. Results show, that, while the technological premises of distance learning can be accommodated by hybrid satellite/wireless infrastructures, this has to be complemented with (a) high-quality audiovisual educational material, and (b) the opportunity for community members to interact with other community members either as groups (common-room oriented scenarios) or individuals (home-based scenarios), thus providing an impetus for learner engagement in both formal and informal activities.
\end{abstract}

Keywords Satellite communications education . Delivery architecture

T. Serif · G. Ghinea $(\bowtie) \cdot$ L. Stergioulas · S. Y. Chen

School of Information Systems Computing and Mathematics,

Brunel University, Middlesex, UK

e-mail: george.ghinea@brunel.ac.uk

T. Tiropanis $\cdot$ S. Tsekeridou

Athens Information Technology, Athens, Greece

\section{Introduction}

Distance learning has undergone radical changes in the past decade due to technological advancements [1]. Among a variety of technologies, e-learning is by now a recognised solution to empower community members to engage in distance learning [2]. In particular, it is useful to provide support for isolated user communities to participate in formal and informal activities. Providing such support involves many iterative cycles between the learning solution providers (pedagogical and technological experts) and the communities for the definition of e-learning services, the identification of e-learning scenarios and eventually the finalisation of e-learning platform requirements. In addition, it involves the setup and fine-tuning of communication infrastructures that can provide reach to isolated areas.

In our research, we have started to explore the above issues by identifying, designing and deploying e-learning services over an integrated satellite and wireless broadband infrastructure for isolated agrarian communities in Greece and Cyprus as well as maritime communities on ships. Figure 1 presents the generic network architecture behind our research. The proposed methodology includes the following phrases:

1. Service elicitation: isolated user communities were involved in the process of eliciting services and service scenarios that can empower communication, collaboration and learning. Initial services were identified and potential learning scenarios were outlined.

2. Evaluation: community members were invited to provide detailed feedback on the first compilation of services and service scenarios.

3. Scenario finalisation: based on the outcome of the evaluation phase, it was possible to finalise the 
learning scenarios that combine identified services into learning experiences for the community members.

4. Network and services requirements: the requirements for the performance of the terrestrial wireless and satellite network as well as the communication services for learning were compiled as part of this phase.

5. Deployment and trial: implementing the software and network architecture and running trial sessions to examine the extent to which the service and network requirements are respected and to evaluate the community members' satisfaction with the implemented learning experience.

This paper presents our experiences and results in respect of empowering isolated communities to engage in distance learning by implementing the above methodology. Accordingly, the structure of the paper is as follows: Sect. 2 presents background material related to e-learning environments for distance learning, whilst Sect. 3 details the elearning services for geographically isolated communities that we have considered in our research, as per the service elicitation phase of our methodology. Building on these proposed services and corresponding to the evaluation phase, Sect. 4 details the results of a questionnaire-based survey which further refined the proposed set of services. Section 5 then targets the scenario finalisation phase of the methodology, presenting the resulting design of realistic learning scenarios, while Sect. 6 elaborates upon the identified learning services. Section 7 presents candidate satellite-based network architectures and networking requirements for the delivery of educational content, while Sect. 8 details inter-operability requirements of such systems and experiences with the deployment of one such architecture in practice, corresponding to the last phase of our methodology. Finally, conclusions are drawn and possibilities for future work discussed in Sect. 9.

\section{E-learning environments for distance learning}

E-learning, one of the tools emerged from information technology, has been integrated in many high education institutes [3]. Urdan and Weggen [4] define e-learning as "the delivery of content via all electronic media, including the Internet, intranets, extranets, satellite broadcast, audio/ video tape, interactive TV, and CD-ROM". E-learning thus encompasses all currently available instruments, including digital or electronic tools, to facilitate learning and impart the contents of a course [5, 6]. Urdan and Weggen [4] also define e-learning as a subset of distance learning, as depicted in Fig. 2.

E-learning is a term that nowadays covers almost any type of learning process based on information and communication technologies. E-learning is not just a course converted to be stored in a personal computer; it rather represents a new mix of resources, interactivity and achievements; new structures for learning, a combination

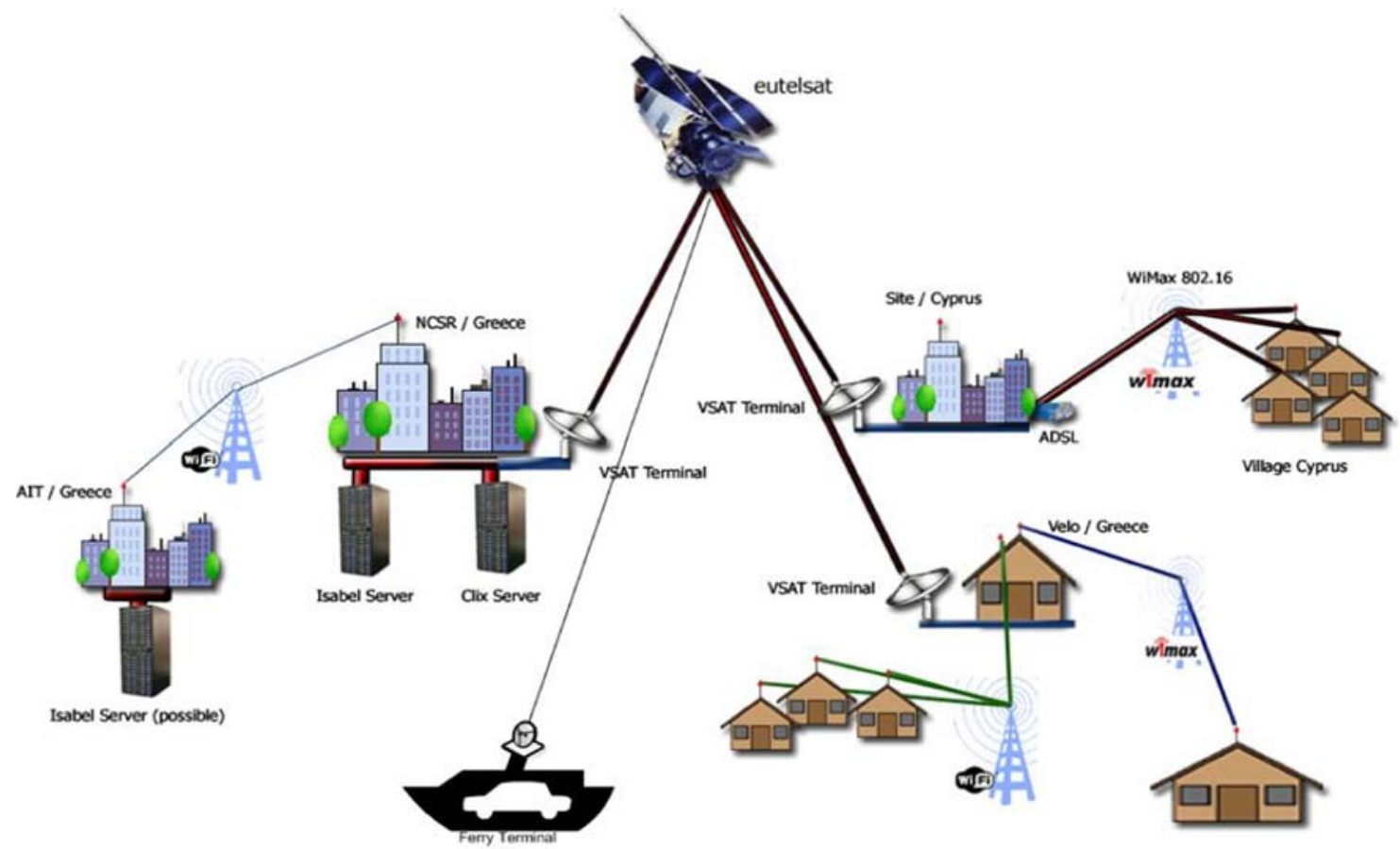

Fig. 1 Generic satellite and wireless network architecture for education 


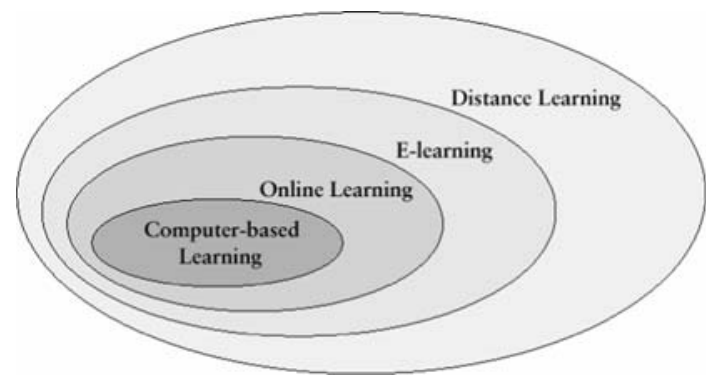

Fig. 2 Subsets of distance learning 0

of teaching services using technological tools that adds value, anytime and anywhere [7].

As depicted in Fig. 3, e-learning is a composition of three main areas: contents, technologies and services [8]. The contents are the didactic units or reusable modules of the e-learning process. The technologies are the technological tools (platforms) that support the educational systems, including communication networks, protocols, and learning management systems among others. The services refer to the tracking and support offered to the students; e.g. tutorials, periodic reports, assessments, course management, student motivation, course quality, etc.

E-learning systems are defined as the platforms or systems offering features that support educative services through electronic media. These systems can be learning management systems (LMS), learning content management system (LCMS), knowledge pools, or brokerage systems $[9,10]$.

- A learning management system is an application running in a web server that is used to perform learning activities. It is also called a platform or virtual campus. It is a place where students, tutors, teachers and coordinators connect through the Internet to download contents, examine the topics of each course, send e-mail to the teachers, chat with students, discuss in a forum, assist to a tutorial, etc.

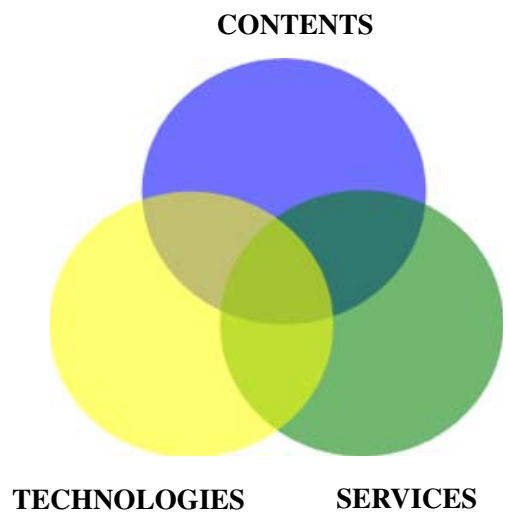

Fig. 3 E-learning components 0
- A learning content management system is a system that is independent or integrated into a LMS. It manages the learning contents. Once the contents are into the system, they can be assigned to each course, combined, downloaded, etc.

- The knowledge pools are distributed repositories of metadata compatible with the learning object metadata (LOM) standard which assure an efficient publication, search and restore of educative contents. An interesting characteristic of these servers is that they can be federated.

- The brokerage systems store the description of the educative resources available locally or at remote locations.

The diversity of the aforementioned systems suggests that e-learning provides multiple functions for educative services, which, in turn, improve the quality of distance learning. This may be one of the reasons why e-learning is popular to students [11] and is regarded as one of the successful factors for distance learning [12]. Nonetheless, there is no recognised methodology for user-led and centred design of satellite-based distance learning architectures to support geographically isolated communities. This is precisely the focus of the paper, the results of which are described in the following sections.

\section{E-learning services for geographically isolated communities}

In keeping with our proposed methodology, a set of services that can be combined in different leading scenarios was first identified in collaboration with selected members of the agrarian and maritime communities who had more experience with the training requirements of their communities.

During this phase a list of services were presented to the representatives of the two communities who were able to give initial feedback on whether they should be further considered and offered for evaluation via questionnaires to the who of the community. In addition, the representatives of the agrarian and maritime communities were able to provide some hints on the different modes of operation that could be considered for the identified services. The community representatives in both cases were community members with more advanced knowledge in ICT than the average community member. Therefore, they were able to collaborate efficiently both with the technological partners in the project and their communities. In addition, the number of users who provided evaluation feedback on the initial scenarios had a higher familiarity with ICT than the average community members; they are thus expected to 
become the catalysts for enabling education in isolated communities in the project.

Although previous projects [13] have succeeded in providing satellite-based education in isolated areas in Greece, the learners involved had had a satisfactory background in ICT. Moreover, such work had not undertaken any detailed service elicitation, nor had it scoped out requirements in respect of the number of services to be deployed and their features. All are issues that we have addressed in our work; indeed, the low familiarity with ICT has been an important challenge.

Following our approach detailed in Sect. 1, as a result of the service elicitation phase, the following services were considered for evaluation [14].

- Virtual classroom (VC) service: was favoured as a candidate service for evaluation by community members. A VC is defined as a computer accessible, on-line learning environment intended to fulfil many of the learning facilitation roles of a physical classroom.

- Learning management system (LMS) service: among the services that were also identified in $\mathrm{BASE}^{2}$ as relevant by the community members was the deployment of a learning management service (LMS) for individual access to educational material anywhereanytime.

- Tele-conferencing service: is a core service for the BASE $^{2}$ vision and its value was recognised by the community member representatives. It enables live sessions connecting many geographically dispersed sites, either ad-hoc or prearranged.

- Webinar/webcast service: consists of a unidirectional, live or pre-recorded, transmission of content from one site to other remote sites. The date and details of each session needs to be announced in advance in order for the receiving site to tune in properly.

Services in addition to the ones above, involving the delivery of pre-recorded educational content, either broadcasted over satellite core and access networks at prescheduled times or by the course coordinator, had also been identified as potential candidates. However these turned out not to be top of the priority list compiled by the community members participating in this phase of service elicitation.

\section{User-based evaluation of e-learning services in the communities}

Following the initial identification of services as candidates for further evaluation user questionnaires were authored to elicit feedback from a much larger base of the agrarian and maritime communities participating in the project.
Evaluation was made through participants completing a questionnaire. This was comprised of five sections:

- Introduction: this section introduced our research work and the purpose of the questionnaire.

- Participant profile: here demographic data, such as gender, age, marital status, as well as more specific information relating to access to computer resources and computing experience was collected.

- Virtual classroom: this section in the first part provided a generic description of a virtual classroom service and collected generic information on their interest to such services. The second part contained two real-life scenarios (common room access and home access) where virtual classroom service was provided and participants' preferences were recorded.

- Learning content management system: initially LCMSbased learning was described and generic experience and awareness questions were asked. In the following part, two LCMS-based learning scenarios (common room based and home based) were depicted and scenario and content quality related preference responses were recorded.

- Tele-conference: this section provided a brief description of the tele-conference service and collected information on awareness, experience and interest of the participants. Following this, the participants were asked to consider two (common room and home) scenarios and requested to provide their preferences on which scenario they were more interested, how they would like to access this service and ideal content sharing modalities of such service.

- Webinar/webcast: this section describes the analogy of webcasts/webinars and to which communities it can serve better. Consequently, the interest and intention of use of the participants was recorded. In the following part, common room based and home based use of this service was described and interest to a specific use, scheduling of the service and usability, and content sharing modality questions were asked.

- Other services: finally, this section described the broadcast and multicast service to common rooms and collected their preferences on the content they were interested in, access methods (PCs/projectors), possible frequency of their attendance to this service and the nature of sessions (moderated/non-moderated).

A total number of 76 respondents, 27 maritime and 49 agrarian users, completed the questionnaire. Maritime users were employees of the SuperFast Ferries and the Minoan Lines companies, on ships that also undertake international routes. Agrarian users were gathered from distributed geographic locations in Greece, to measure differentiating user interests and needs per regions. 
Seventy-six percent of the users were men and $24 \%$ were women. Most of the users (61\%) were in the 35-49 age range, while the rest were mainly in younger age brackets. Although the overwhelming majority (89\%) of surveyed users are accustomed to technology and electronic communication, members of both communities do not currently get the chance to participate in training programmes, attending educational seminar programs only a few times a year.

The questionnaire also revealed that almost $90 \%$ of the members of both communities have never participated in a teleconference. Nevertheless, $54 \%$ of the participants in this survey are very or extremely interested in participating with colleagues from remote areas in future, while a significant $32 \%$ appears to be open to this possibility. One in four of the participants consider it very or extremely important to be able to use TV-based equipment instead of a PC for participating in video conferences (as shown in Fig. 4) which is indicative that the motivation factor needs to be strengthened for participation in virtual classroom services. Almost none of the participants have had the experience of participating in a Webinar/Webcast session. However, half of them are very or extremely interested in participating in Webinars, while more than one in three expressed that they are open to this potential. One in three of the members of the maritime community consider very or extremely important to be able to participate in Webinars using TV-based equipment, while one in four members of the agrarian community place high importance on this feature.

For both communities, three out of four participants had never yet used a learning management system nor an educational portal to access education resources. Nonetheless, $61 \%$ of respondents would be eager to use such a system with 50\% stating that they would also be using the LMS system for collaboration with other colleagues and exchange of ideas. Moreover, $62 \%$ of users believe communication with remote tutors and learners to be of great importance. The preferred modes of remote communication

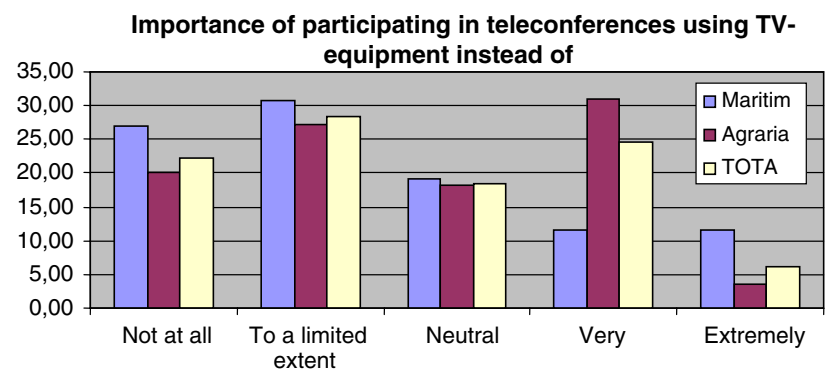

Fig. 4 Preference for TV-based vs. PC-based terminal equipment for education and collaboration are emails (64\%), chat (50\%) and forums $(44 \%)$.

When presented with the options of receiving low quality video streaming of educational lectures in a timely manner rather than high quality videos but with longer delays, users from both communities are open to adapted implementations per case. Moreover, both communities are in favour of the dynamic adaptation feature of the learning process, believing in its efficiency to provide enhanced learning experiences. Finally, $72 \%$ of users across both communities prefer synchronous services, while $28 \%$ opted for asynchronous ones. As far as the prospect of being provided with integrated services is concerned, that is combining both synchronous and asynchronous types of services, more than half of the users of both communities believe it to be very or extremely important.

\section{Design of realistic learning scenarios}

Given the feedback received via the questionnaires a number of learning scenarios was finalised and the functional and non functional requirements of the identified services were detailed using the Volere template [15]. This is an important phase of the methodology, since, these learning scenarios will form the basis on which the service and network architecture requirements will be identified and trade-offs in terms of how these requirements can be best met will be considered.

Thus, a number of tele-education and e-learning scenarios were defined to present the direct deployment of satellite-based learning services, which will be described in the following sections. Each scenario may involve the deployment of part of a service, of a full service or of a combination of services based on the feedback received during the evaluation, which is precisely the reason why we distinguished between services and scenarios in our work. Further, the specified scenarios present the basis for the last phase of our methodology, namely the trials definition and evaluation of the tele-conferencing services and associated infrastructure.

The scenarios are grouped into two major categories, as illustrated in Fig. 5:

- The common room oriented e-learning scenarios: these address cases where groups of learners are gathered in a common room to be educated using a satellite-based learning service. The key factor here is groups of learners in a common room: that is, a tele-education room is setup with all required equipment to access the distance learning service and access is mainly scheduled and occurs simultaneously. 
- The home oriented e-learning scenarios: these refer to cases where each learner accesses the distance learning services from home, at any time of the day, using his/ her own enabling equipment

(a)

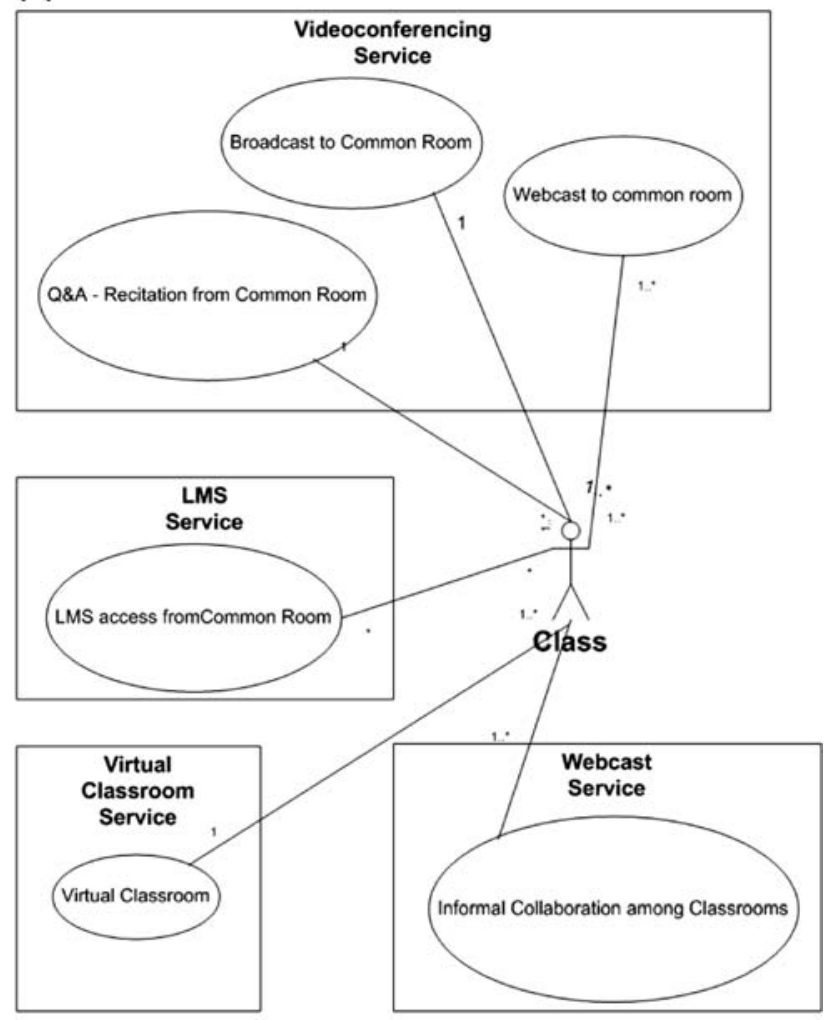

(b)

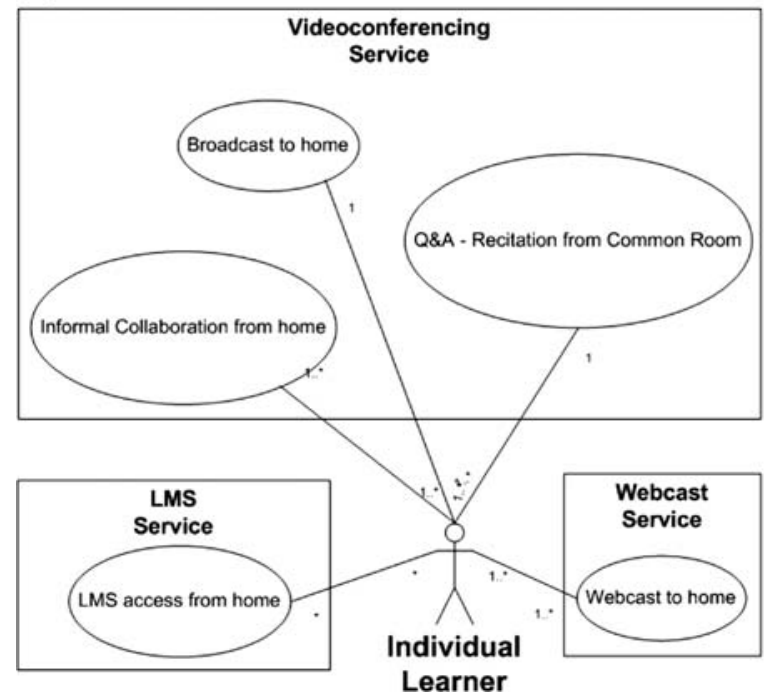

Fig. 5 High level use case diagrams for $\mathbf{a}$ common room and $\mathbf{b}$ home based learning

\section{Satellite-enabled education services}

Based on the collected requirements, a number of useroriented services can be identified.

\subsection{Virtual classroom service}

A virtual classroom is defined as a computer accessible, online learning environment intended to fulfil many of the learning facilitation roles of a physical classroom. It provides a distributed learning environment at any time, any place and at any pace. By using virtual classrooms, one can hold face-to-face conversations and collaborate with coworkers around the world.

\subsection{Learning content management system service}

Among the services that have been identified as relevant to the scope and objectives of the satellite-enabled education is the deployment of an LCMS. This service supports the authoring, publication and management of learning content, including multimedia content adaptation aspects, from the viewpoint of the system administrator and author but at the same time it will provide for personalised access to combined learning objects (LOs) in a learning path that suits best the learners of the participating communities.

\subsection{Tele-conferencing service}

This is a core service enables live sessions connecting many geographically dispersed sites, either ad-hoc or prearranged manner. The functionality offered by a teleconferencing service is similar to that of the virtual classroom, however it is more focused on live interaction and more flexible with regard to time scheduling and site participation, but with limitations in terms of delivering materials. Tele-conferencing can be defined as bi-directional communication between two or more groups, or between three or more individuals, who are in dispersed sites.

\subsection{Webinar/webcast service}

This service consists of a unidirectional, live or prerecorded, transmission of content from one site to the rest of remote sites. The date and details of each session should be announced previously in order for the receiving site to tune in properly. 
The main actors that interact in the educational process could be teachers and learners, or just learners among them. There are no restrictions on the communities that could be able to use this service; in principle any community (including agrarian and maritime) can benefit from it.

While the content can be pre-recorded at the transmitting site, this service does not involve on-demand download. In that way the receiving site can not distinguish if the content being played is live or pre recorded. The only difference in this case is at the transmitting site. The content could be received at a common room or at the learner's home. Both scenarios are pretty similar. The main difference is the need for a more sophisticated common room setup. It is expected to use common-of-the-shelves player software at the receiving site.

\subsection{Other services}

Another potential service that accommodates the needs of already defined e-Learning scenarios is the "Broadcast/ multicast e-Learning service". In such a service, prerecorded educational content is either broadcasted over satellite core and access networks (DVB-S2, VSAT) or multicasted over broadband satellite and wireless IP-based access networks (DVB-RCS, Wi-Fi, WiMAX) respectively to remote groups of learners gathered in a tele-education hall, equipped with receiving satellite antennas and hubs or wireless access points, set-top boxes or PCs, video projectors, VCRs, TV sets, microphones and speakers. The session is initiated either at pre-scheduled times or by the course coordinator, and it involves, initially passive viewing of the educational course and later on communication and interaction possibilities for Q \& A sessions.

\section{Satellite-network architecture for distance learning}

The rationale in supporting multiple satellite communication environments stems from: (1) the variability of expected user requirements (some users have inflexible legacy communication systems, while others can be open for very innovative deployments), and (2) the existence of a number of satellite platforms with a wide range of capabilities and cost. Accordingly, we consider three distinct satellite network architectures for education applications, which we now proceed to describe.

\subsection{VSAT architecture}

\subsubsection{Land-to-L and VSAT architecture}

This architecture includes a number of VSAT enabled sites and further interconnection using $\mathrm{WiFi}$ and/or WiMax wireless infrastructure for delivering the service to more users (Fig. 6). Different usage scenarios can involve pointto-point, point-to-multipoint interactive communication, live lectures and video on demand capabilities, as well as interconnection between the network sites in order to ensure the necessary collaboration between the learning groups, which is an essential e-learning element.

In the VSAT architecture, each VSAT station has its own distinct purpose. A central VSAT is needed for the broadcast of the live lectures using a fully equipped studio and the provision of the educational offline material from the e-learning platform. This VSAT station acts as a central node in the process of educational content consumption. Another VSAT antenna provides the e-learning service to users attending the course live at the tele-education hall or through a WiFi connection from other premises. The third

Fig. 6 VSAT networks

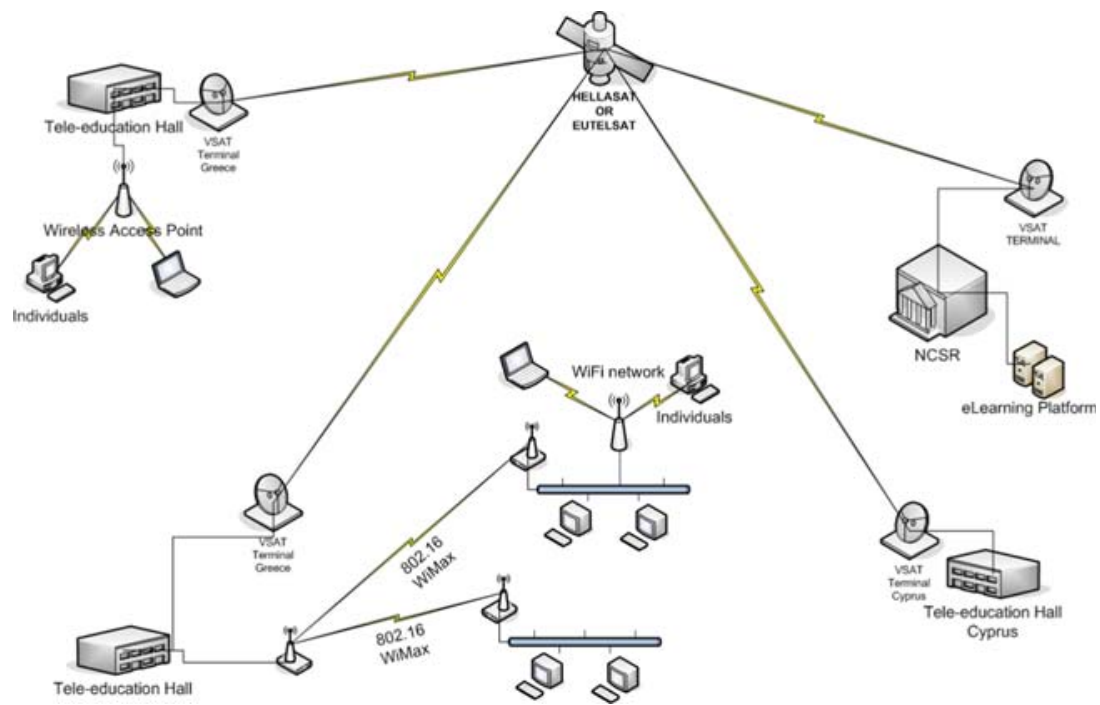


VSAT antenna is used to facilitate the testing of VSAT and WiMax in combination with the WiFi networking. The VSAT network will have mesh topology and thus will provide the possibility of collaboration among sites.

\subsubsection{Land-to-vessel VSAT architecture}

This network architecture includes one or more ship(s) connected with a VSAT station at the central node with a fully equipped studio (Fig. 7). It facilitates live lectures and video on demand capabilities using the IPv4 protocol. In order to realise the proposed e-Learning services on VSAT networks, our tests have revealed that we require a minimum of 500-550 ms round trip time (RTT) on a 1.8-2.4 m antenna with $8 / 25 / 40 \mathrm{~W}$ SSPA/TWTA. The required

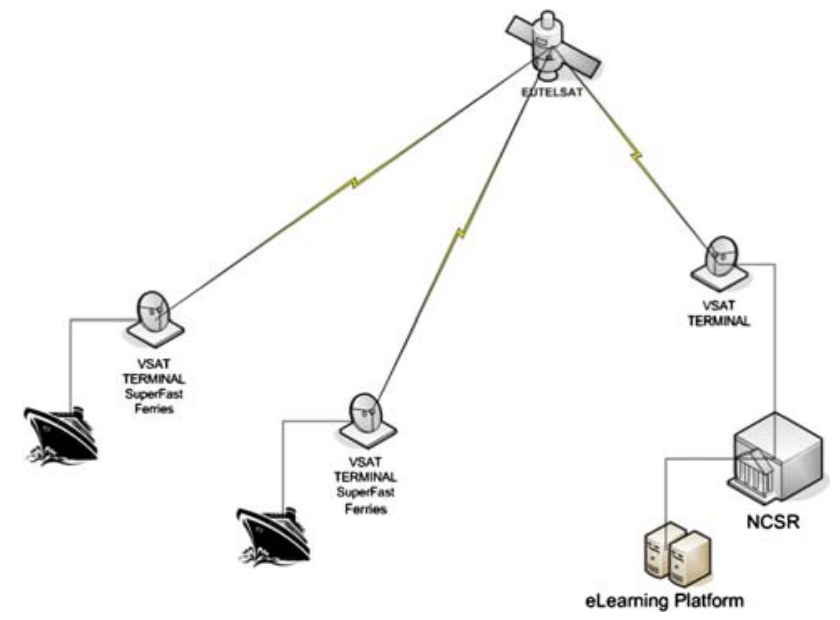

Fig. 7 Maritime network structure

Fig. 8 DVB-S/S2 network structure satellite bandwidth for the network would be approximately $11.5 \mathrm{MHz}$ for an $8 \mathrm{Mbp}$ shared mesh carrier, with a bit-error-rate (BER) equal to or less than $10^{-8}$, well within the boundaries for user acceptance [16].

\subsection{DVB-S/S2 architecture}

This architecture involves DVB-S or DVB-S2 enabled sites for e-learning. It can support e-learning scenarios, such as point to point and point to multipoint interactive communication and collaboration, as well as live lectures and video on demand capabilities using the IPv4 and IPv6 protocols (Fig. 8). In a DVB-S/S2 architecture, the quality of service (QoS) requirement are quite different to those of VSAT's. Accordingly, here the expected round trip time should be in the range of 250-280 ms unidirectional and $300-2,000 \mathrm{~ms}$ bidirectional (with terrestrial return). The dish at the hub station should be 3.7-9 m with 100+ Watts TWTA output and at the receiver end 2.4-6 m. Depending on the technology used, network bandwidth could reach up to $120 \mathrm{Mbp}$ in the range of $1.5-36 \mathrm{MHz}$. In order to achieve a smooth e-learning content transmission the overall bit-error-rate should be less than $10^{-9}$. Again, this has been shown to be well within user acceptance limits for such applications [16].

This architecture involves DVB-RCS enabled sites connected with the central DVB-RCS platform (Fig. 9). Similarly to the previous architecture, this architecture can support live lectures and video on demand capabilities using IPv4 protocol. The DVB-RCS architecture strictly follows the approach of a star distribution system with one outgoing broadband satellite channel from a central hub station and multiple return channels originating from

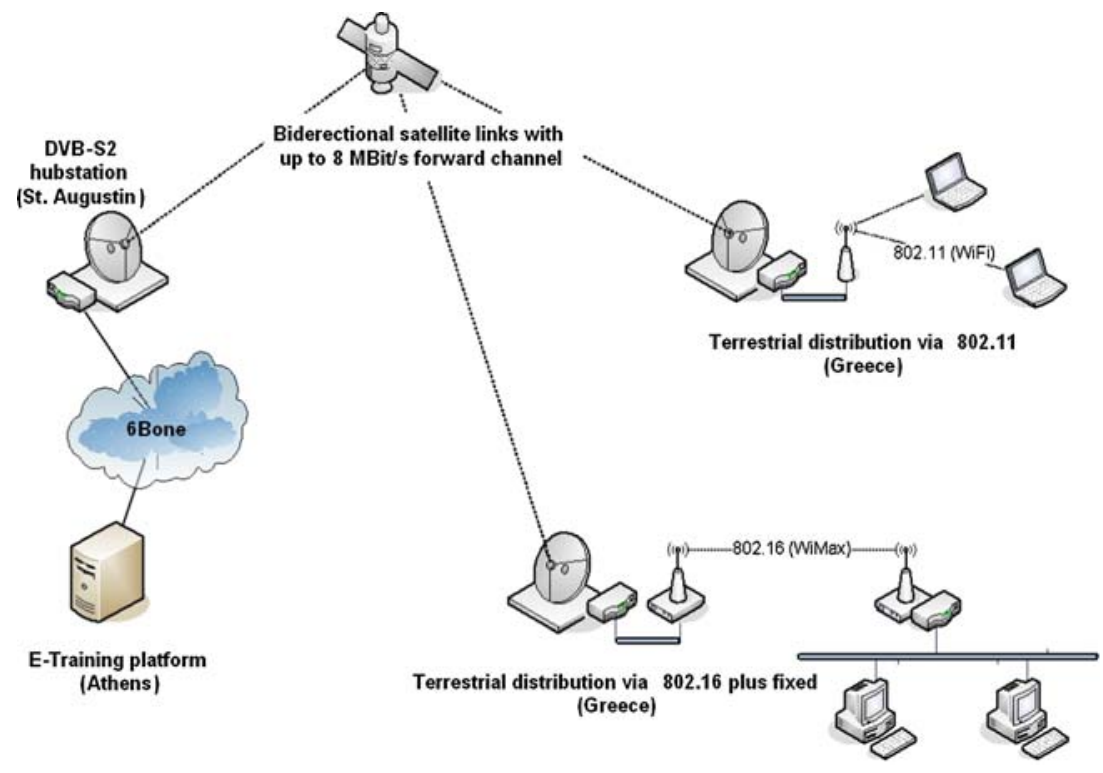


VSATs which log-in to a network management system via the satellite link. The return channel architecture is organized in MF-TDMA time slots in which either MPEG-2 or ATM cells are utilised. In order to guarantee a quality transmission of e-learning services on such an infrastructure, the expected round trip time should be in the range of 580-650 ms and overall bit-error-rate less than $10^{-9}$. Such parameter values are again well within user acceptable boundaries [16]. In this configuration, the dish at the hub station should be 3.7-9 m with 100+ Watts TWTA output and at the remote station $0.98-2.4 \mathrm{~m}$ with 1-4 W BUC SSPAs. The bandwidth for the hub station would be 1.5$45 \mathrm{Mbp}$ at $1.5-36 \mathrm{MHz}$, and for the remote stations $0.016-$ $2048 \mathrm{kbp}$ at $0.02-2.2 \mathrm{MHz}$.

\section{Deployment and trial of the software and network architecture}

As part of the deployment and trial phase: the last phase of our methodology: an assessment of the integrated hybrid network infrastructure, which brings together the broadband terrestrial networks and satellite communication technologies, was undertaken using as input the required QoS and technical parameter values detailed in Sect. 7.

At this stage in our work, we have concentrated on the DVB-RCS architecture described above, as it was considered important to be able to afford users the possibility of a return channel; moreover, this was to be done via satellite as the terrestrial infrastructure could very well be limited in geographically isolated communities. In this context, two deployment scenarios have been considered. While the first comprises multiple satellite terminals and explores the possible use of the satellite based distance learning system by multiple users concurrently, the other integrates satellite, wireless and ethernet technologies; both shall now be described.

\subsection{Deployment scenarios}

\subsubsection{Scenario 1: DVB-RCS (three sites to one Router)}

This scenario consists of four PC-class terminals. Three of them were attached to remote DVB-RCS satellite interactive terminals, and the fourth was attached to the DVBRCS HUB station. As shown in Fig. 10 the uplink and downlink data rates for the three sites (SIT1, 2 and 3) are $1024 \mathrm{kbp}$.

\subsubsection{Scenario 2: DVB-RCS-WiFi (one site)}

This scenario consists of two PC-class terminals. One is attached to a remote EMS DVB-RCS satellite interactive terminal via $\mathrm{WiFi}$, and the other was attached to the SAT DVB-RCS HUB station. As shown in Fig. 11 the uplink and downlink data rates for SIT1 $1024 \mathrm{kbp}$.

\subsection{Evaluation strategy}

The two scenarios were then assessed in two phases: the first examined the communication and interoperability of the technologies that make up the hybrid tele-education network and tested for any unwanted jitter and delay. The second weighed up the transmitted content against the
Fig. 9 DVB-RCS network structure

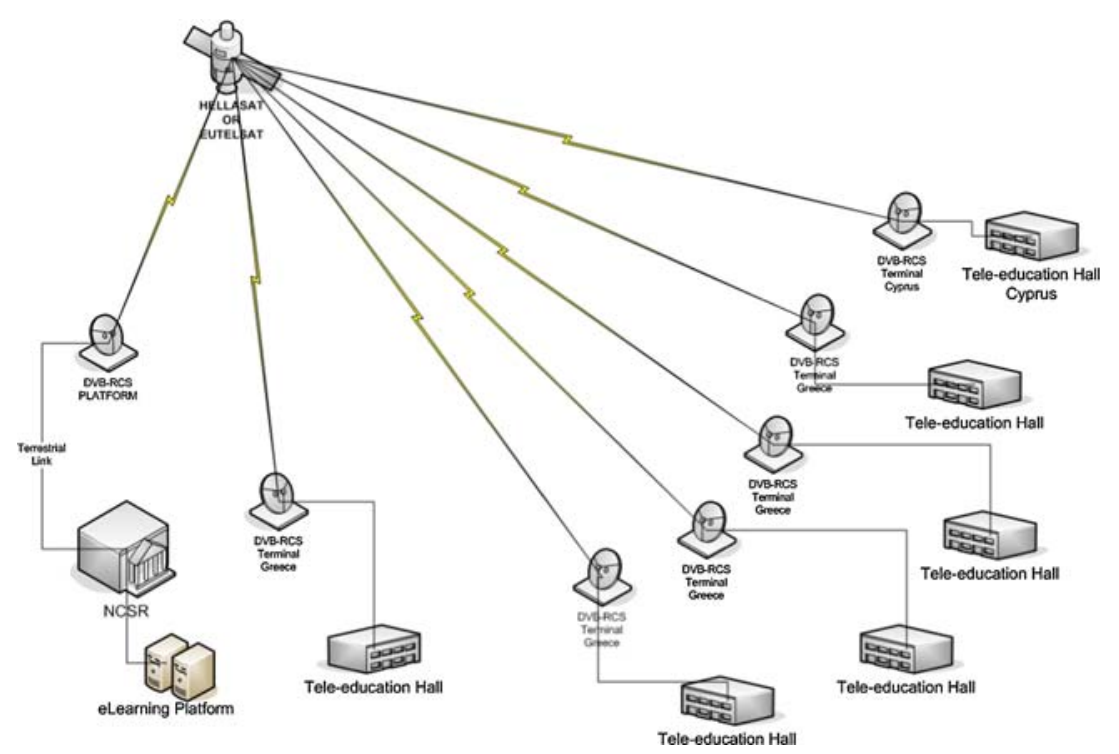


Fig. 10 Scenario 1: DVB-RCS (three sites)

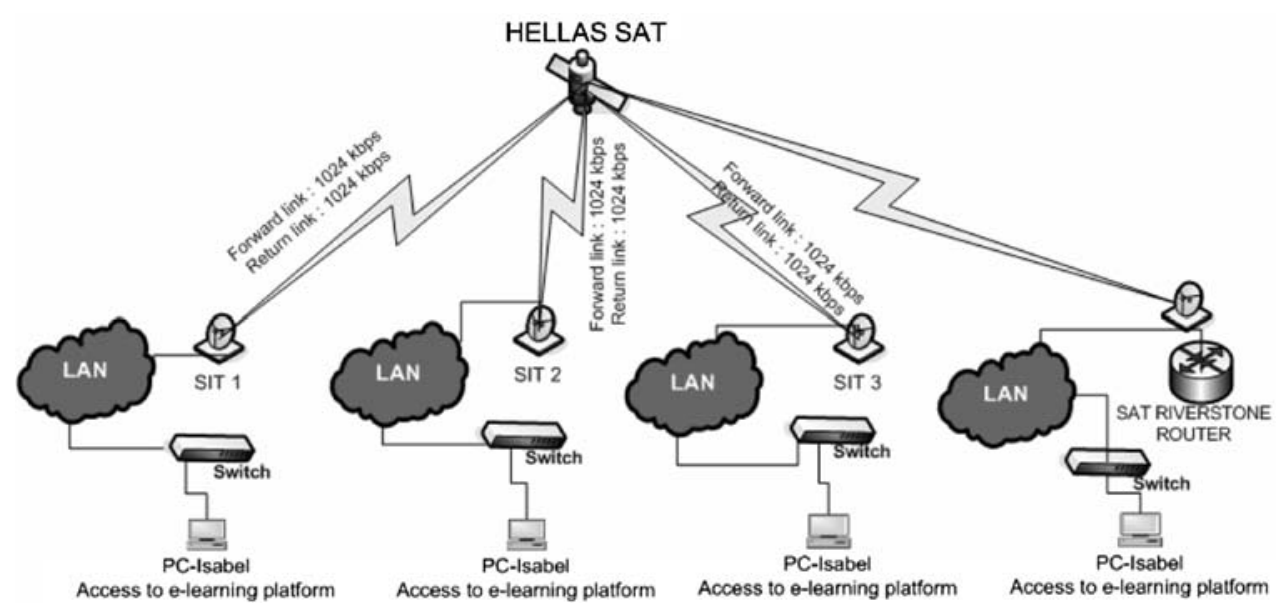

Fig. 11 Scenario 2: DVBRCS-WiFi (one site)

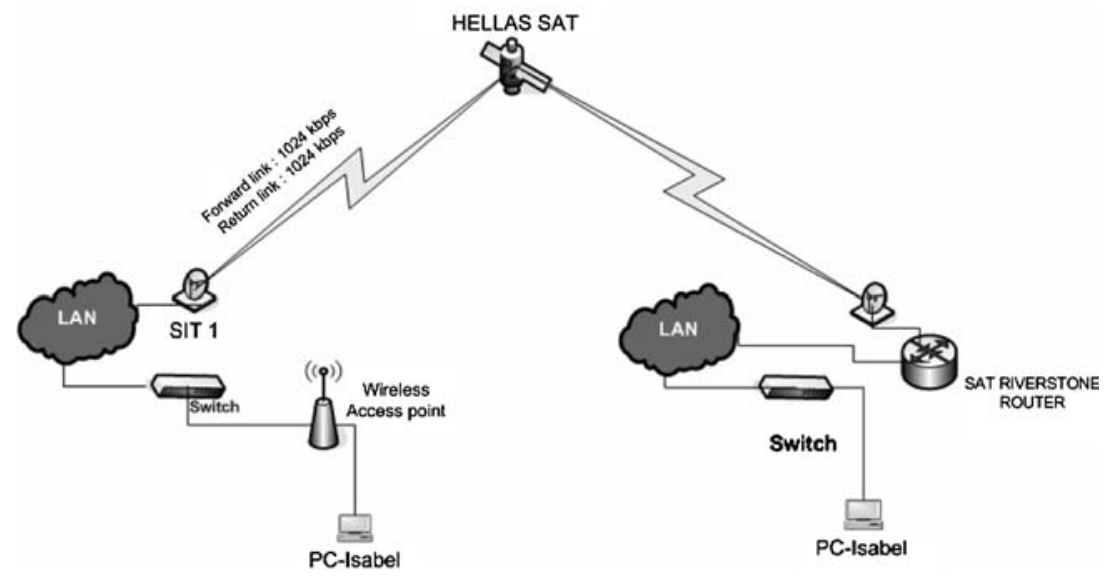

QoS requirements, and further evaluated the learner/user experience.

\subsubsection{Connectivity and interoperability}

The first phase aims to evaluate the overall performance of the hybrid network by measuring the roundtrip delay, packet loss, and availability between hosts.

Roundtrip delay is the interval between the time a measurement application sends a packet to a network host and the time it receives an acknowledgment that the packet was received by the this particular host. Roundtrip delay also includes any queuing delays at the endpoints or the intermediate hosts. In our trials, the ping network tool, which uses the Internet Control Message Protocol (ICMP) Echo function detailed in RFC 792, will be utilized.

Packet loss is the fraction of packets sent from a measurement network tool to a test host for which the measurement tool does not receive an acknowledgment from the test host. In our trials, the Iperf network tool, which enables to measure maximum TCP and/or UDP bandwidth by allowing the tuning of various TCP parameters and UDP characteristics, will be used. This network tool also reports on delay jitter and datagram loss of a specific link between to network hosts.

Network availability testing is the availability measurement between two network hosts at a given time. If the measured packet loss rate and the relevant statistics on the roundtrip delay (such as median or maximum) are below predefined thresholds the network can be defined as available.

If our hybrid network successfully exceeds the connectivity and interoperability requirements, the trials will proceed to the next phase.

\subsubsection{Transmission QoS and user experience}

To examine the network QoS parameters a network performance meter (netmeter) was used to monitor the communication between the server and the learner community users. This application provides an integrated graphical interface and abundance of tools that allow the measurement of QoS values over IPv4 and IPv6 networks. The captured log files are then compared with requirements 
Table 1 Quality of service parameters for an acceptable audio/video delay (based on 0)

\begin{tabular}{lll}
\hline Media & Mode/application & $\begin{array}{l}\text { Quality } \\
\text { of service }\end{array}$ \\
\hline Video & & \\
Animation & Correlated & $\pm 120 \mathrm{~ms}$ \\
Audio & Lip synchronisation & $\pm 80 \mathrm{~ms}$ \\
Image & Overlay & $\pm 240 \mathrm{~ms}$ \\
& Non-overlay & $\pm 500 \mathrm{~ms}$ \\
Text & Overlay & $\pm 240 \mathrm{~ms}$ \\
& Non-overlay & $\pm 500 \mathrm{~ms}$ \\
Audio & & \\
Animation & Event correlation (e.g. dancing) & $\pm 80 \mathrm{~ms}$ \\
Audio & Tightly coupled (stereo) & $\pm 11 \mu \mathrm{s}$ \\
& Loosely coupled (dialogue mode & $\pm 120 \mathrm{~ms}$ \\
& $\quad$ with various participants) & \\
& Loosely coupled & \\
& $\quad$ (e.g. background music) & \\
Image & Tightly coupled & (e.g. music with notes) \\
& Loosely coupled (e.g. slide show) & $\pm 500 \mathrm{~ms}$ \\
& Text annotations & $\pm 240 \mathrm{~ms}$ \\
Text & Audio related to the item & $-500 \mathrm{~ms},+750 \mathrm{~ms}$ \\
& to which the pointer shows & \\
\hline
\end{tabular}

compiled based on the user experience literature [17] [18] (Tables 1, 2).

\subsection{Results and analysis}

\subsubsection{IP unicast test}

The aim of the IP unicast test is to study the links connectivity and also measure its delay, thus addressing the first phase of the evaluation strategy highlighted in the previous sub-section. For scenario 1, the three sites links connectivity was determined by looking at packet loss ratios for all the links. Figure 12 shows that for this pretrial test the 50 packets that have been exchanged among the various sites in this scenario have been almost delivered successfully with $0 \%$ packet loss except for PC2 packets. This proves that the network connectivity for this scenario has been maintained efficiently.

For delay and jitter measurements, results show that the total round trip time is maintained within the range of 5,000 ms, and Fig. 13 shows the total and average RTT for all paths in the scenario. For RTT total, 5,000 ms is considered to be a significant amount of delay, due to the fact that satellite links introduce a considerable time delay value. With regards to RTTavg, the figure shows delay variation among the paths ranging from figures as small as $670 \mathrm{~ms}$ up to $2,200 \mathrm{~ms}$.

On the other hand, scenario 2 attempts to study connectivity and delay issues for satellite and WiFi topology. In terms of connectivity, our trials revealed loss rates of less than $10^{-10}$, highlighting that the network connectivity for this scenario has been maintained efficiently. However, with $\mathrm{WiFi}$, mobility is considered to be an important issue for measuring time delay. Figure 14 examines whether mobility will have an effect on delay measurements. At this point the client: a mobile laptop: connected via WiFi to the $\mathrm{PC} 1$, moved outside the range of WiFi coverage and time delays increased up to $1 \mathrm{~s}$. the mobile client then returned within the WiFi range and time delays returned to normal satellite average delays of approximately $600 \mathrm{~ms}$.

\subsubsection{ISABEL}

The Isabel CSCW application is a group collaboration tool for the Internet (or other IP networks), which uses TCPUDP/IP protocols (IPv4, IPv6 and dual stack). Isabel supports the realization of distributed meetings, classrooms, congresses, etc., by using a service concept which has a very effective management of multipoint configurations.

The Isabel service concept tries to develop the idea that collaboration over the network must follow the same rules as if the participants are sharing the same physical location. Accordingly, Isabel services are designed to create a collaboration environment that enables its users to interact with other participants as if they are taking part to a group activity in a physically shared location. Moreover, each
Table 2 User acceptable loss for audio/video content (adapted from 0 )

\begin{tabular}{|c|c|c|c|c|}
\hline & \multicolumn{4}{|c|}{ Probability of losing frames } \\
\hline & \multicolumn{2}{|l|}{ Audio } & \multicolumn{2}{|l|}{ Video } \\
\hline & $\begin{array}{l}\text { Task dependent } \\
\text { ratings }(\%)\end{array}$ & $\begin{array}{l}\text { Task independent } \\
\text { ratings }(\%)\end{array}$ & $\begin{array}{l}\text { Task dependent } \\
\text { ratings }(\%)\end{array}$ & $\begin{array}{l}\text { Task independent } \\
\text { ratings }(\%)\end{array}$ \\
\hline Good & 0 & $<4$ & 0 & 19 \\
\hline Still acceptable & 10 & 6 & 99 & 51 \\
\hline $\begin{array}{l}\text { Poorest quality } \\
\text { (slight below acceptable) }\end{array}$ & 22.5 & 10 & & 64 \\
\hline
\end{tabular}




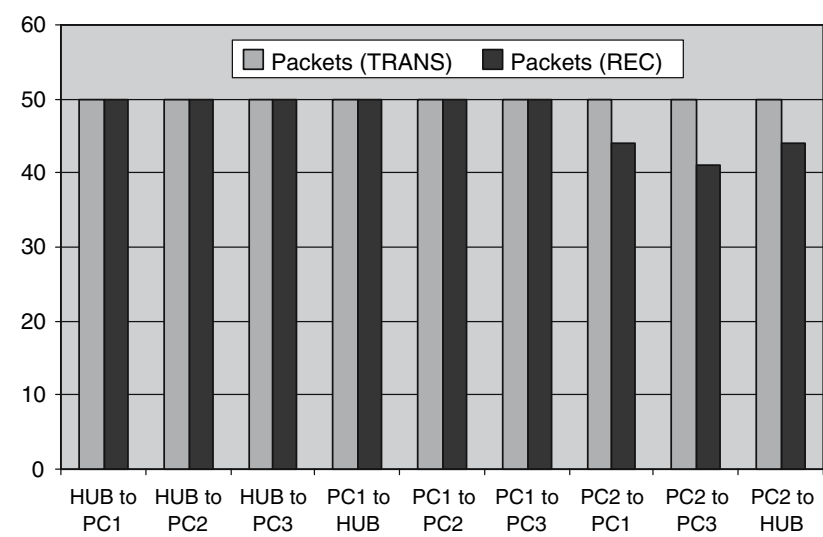

Fig. 12 Scenario 1: DVB-RCS (three sites) transmitted and received packets

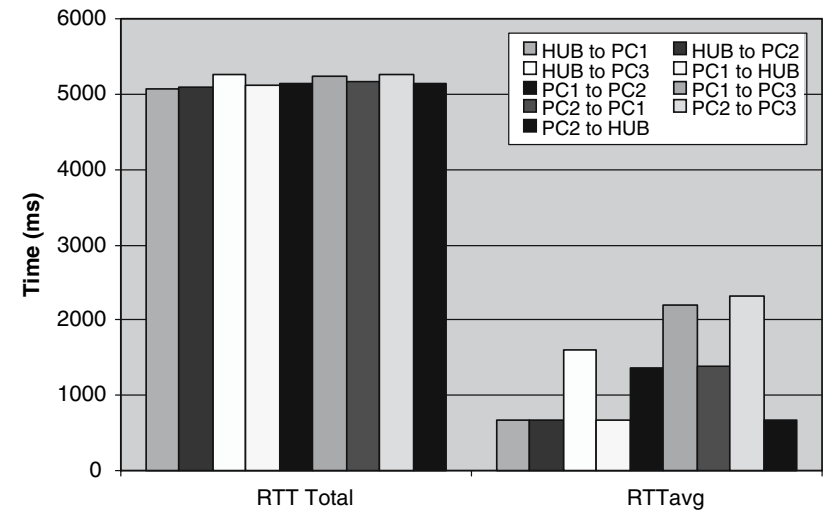

Fig. 13 Scenario 1: DVB-RCS (three sites) round trip times

service has particular configurations of the media to support each type of interaction, which are called "interaction modes".

- A service will have therefore a set of associated "interaction modes", which cover all the type of activities envisaged in it. Examples of interaction modes are chairmen introducing speaker, talk, presentation with slides, demo, application sharing, distributed panel with questions, highlighted speaker with remote audience, etc.

- Each "interaction mode" has an associated visual message which selects only the active sites or highlights them clearly from the inactive ones.

On the other hand, Isabel floor control supports the rules by which the activity is usually managed and has two levels:

- The first level controls the "interaction mode" selection and may vary from the fully centralized model to the fully distributed model, having some intermediate variants.

- The second level provides the control of the components of a given "interaction mode" if any. For example, the request and selection of a question in

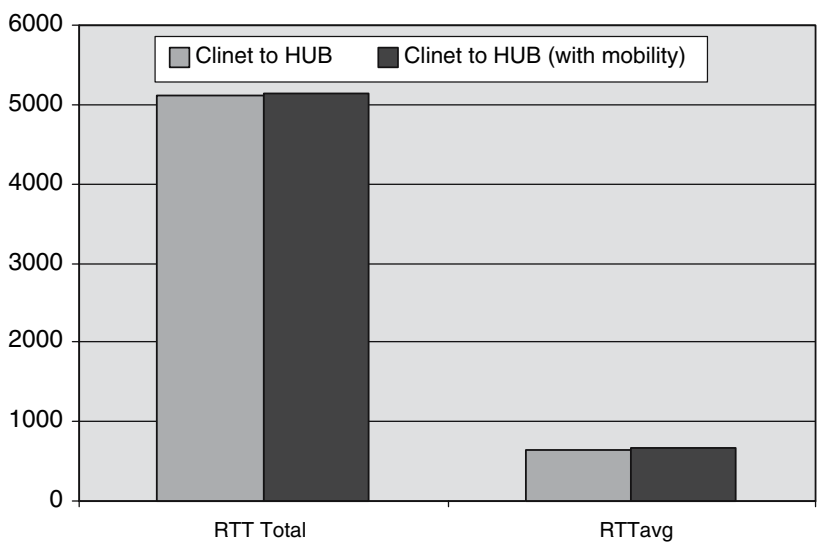

Fig. 14 Scenario 2: DVB-RCS-WiFi (one site) round trip times with and without mobility

the question mode, the access to the blackboard controls or the control of the pointer/pencil in the presentation or demo mode.

A final feature of the Isabel service concept is that all participants always have the same vision or perception of the distributed event. Therefore, any change made (with any of the control panels) reconfigures the presentation of the media in all the sites participating in an event, such that all participants are seeing always the same screen configuration.

\subsubsection{ISABEL tests}

Isabel teleconferencing tool performance tests were conducted using both network scenarios. In order to measure the traffic characteristics, we created a real-life conference environment for each one of the scenarios. At the same time, we ran package capturing applications on the both ends of the network and recorded the whole teleconferencing session, which was $2 \mathrm{~h}$ long.

We split the captured packages into four distinct groups, namely audio, video, other UDP traffic and TCP signalling (Fig. 15). Accordingly, the analysis of the result packages showed that the highest package loss occurred in UDP traffic between the HUB station and the PC terminals (1$3)$. However, there were no significant package losses in audio video traffic between the HUB station and the PC terminals. Overall, audio and video package loss was in the range of 0.16 and $0.14 \%$, respectively, which clearly shows that the results are acceptable for learners.

\section{Conclusions}

Delivering educational content to remote, geographicallyisolated, communities is one of the main attractions of e-learning; however it is one which is rarely implemented 


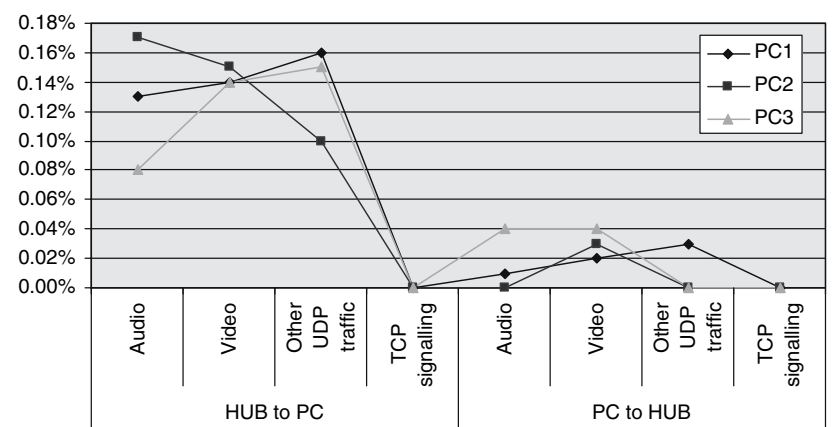

Fig. 15 Scenario 1: loss percentages between HUB and PC terminals

in practice. Moreover, there is no agreed-upon methodology for doing this. This paper has presented our experiences in implementing a hybrid satellite/wireless elearning project in Greece and Cyprus, based upon a userled and -centred methodology. Our results so far have been encouraging, and highlight that while the technological infrastructure can certainly deliver e-learning content well within acceptable perceptual boundaries, appropriate attention must be given to the terrestrial network configuration, as well as to user expressed needs for both formal as well as, importantly, informal engagement with the learning process. We realise, though, the limitations of context in the application of our methodology and we therefore welcome feedback from researchers undertaking similar efforts elsewhere.

Acknowledgments Part of this work was carried out under the auspices of the Aeronautics and Space Programme of European Commission, as part of the $\mathrm{BASE}^{2}$ project (Contract no.: 516159).

\section{References}

1. Tuckerman BW (2007) The effect of motivational scaffolding on procrastinators' distance learning outcomes. Comput Educ 49(2):414-422

2. Sanz S, Iskander MF, Yu L (2000) Development of an interactive multimedia module on antenna theory and design. Comput Appl Eng Educ 8(1):11-17
3. Selm HM (2007) Critical success factors for e-Learning acceptance: confirmatory factor models. Comput Educ 49(2):396-413

4. Urdan T, Weggen C (2000) Corporate E-learning: exploring a new frontier: http://www.spectrainteractive.com/pdfs/ CorporateELearingHamrecht.pdf (Accessed on 3 April 2006)

5. Hall B (2000) New study seeks to benchmark enterprises with world-class elearning in place. E-learning 1(1):18-29

6. Gotschall M (2000) E-learning strategies for executive education and corporate training. Fortune 141(10):S5-S59

7. Kaplan-Leiserson E (n.d.). Glossary. Learning circuits. Retrieved 25 May 2007, from http://www.learningcircuits.org/glossary.html

8. Henry P (2001) E-learning technology, content and services. Education and Training. Emerald Insight 43(4/5):249-255

9. McCrea F, Gay RK, Bacon R (2000) Riding the big waves: a white paper on B2B e-learning industry. San Francisco: Thomas Weisel Partners LLC. McLellan, 1997

10. IEEE, Learning Technology Standards Committee, Learning Object Metadata Standards. Retrieved 29 May 2007, from http://ltsc.ieee.org/wg12/index.html

11. Weller M (2007) The distance from isolation: why communities are the logical conclusion in e-learning. Comput Educ 49(2):148159

12. Papp R (2000) Critical success factors for distance learning. Paper presented at the Americas conference on information systems, Long Beach

13. Sotiriou S, Orphanakis M, Pyrini A, Savas S, Tsolakidis C, Sotiriou M, Tsiopoulos E, Prevedourou D, Tiropanis T, Mpithas S, Prevedouros G, Tavlaki E, Agapiou G, Nikoyiannis A (2004) EDEN (European Distance and E-Learning Network).In: Conference-New challenges and partnerships in an enlarged European Union, Budapest

14. Serif T, Stergioulas L, Ghinea G, Moatsos M, Makropoulos C, Tsekeridou S, Tiropanis T (2006) Satellite-enabled interactive education scenarios and systems architectures. In: Proceedings of the 1st European conference on technology enhanced learning, LNCS, Springer, Crete

15. Robertson J, Robertson S (1995) Volere: requirements specification template. Edition 10, Atlantic Systems Guild. http://www. systemsguild.com/GuildSite/Robs/Template.html

16. Ghinea G, Thomas JP (2000) Impact of protocol stacks on quality of perception. In: Proceedings of IEEE international conference on multimedia and expo, vol 2, New York, pp 847-850

17. Kawalek J (1995) A user perspective for QoS management, In: Proceedings of 3rd international conference on intelligence in broadband services and network (IS ans N '95, Crete, Greece)

18. Blakowski G, Steinmetz R (1996) A media synchronization survey: reference model, specification, and case studies. IEEE J Sel Areas Commun 14(1):5-35 